\title{
(2) OPEN ACCESS \\ Collaborative, patient-centred care model that provides tech-enabled treatment of opioid use disorder via telehealth
}

\author{
Rebekah Rollston, ${ }^{1}$ Winifred Gallogly, ${ }^{2}$ Liza Hoffman, ${ }^{3}$ Eshan Tewari, ${ }^{4}$
} Sarah Powers, ${ }^{2}$ Brian Clear ${ }^{5}$

${ }^{1}$ Research \& Development, Bicycle Health Inc, Boston, Massachusetts, USA

${ }^{2}$ Patient Services, Bicycle Health Inc, Boston, Massachusetts, USA ${ }^{3}$ User Experience Research, Bicycle Health Inc, Boston, Massachusetts, USA

${ }^{4}$ Product, Bicycle Health Inc, Boston, Massachusetts, USA

${ }^{5}$ Clinical Medicine, Bicycle Health Inc, Boston,

Massachusetts, USA

\section{Correspondence to}

Dr Rebekah Rollston, Research \& Development, Bicycle Health Inc, Boston, MA 02111-1995, USA; rebekah@bicyclehealth. com

Received 10 August 2021 Accepted 2 February 2022

Check for updates

(C) Author(s) (or their employer(s)) 2022. Re-use permitted under CC BY-NC. No commercial re-use. See rights and permissions. Published by BMJ.

To cite: Rollston $R$, Gallogly W, Hoffman L, et al. BMJ Innov Epub ahead of print: [please include Day Month Year]. doi:10.1136/ bmjinnov-2021-000816

\section{INTRODUCTION}

In 2020, the opioid epidemic in the USA claimed the lives of 186 people each day, a $26 \%$ increase from the year prior. ${ }^{1}$ Per 2020 National Survey on Drug Use and Health data, only $10.16 \%$ of persons with opioid use disorder (OUD) accessed OUD treatment through existing systems in the prior 12 months, while capacity for office-based opioid treatment (OBOT) remained dramatically underused. ${ }^{2}{ }^{3}$ The approach of training more providers to deliver medication for opioid use disorder (MOUD) through existing systems has not addressed this access gap effectively, and the need for a truly biopsychosocial model of care with a focus on reaching unengaged populations has become increasingly clear.

The most broadly accessible existing level of care is the OBOT model. ${ }^{4}$ The foundation of this model is the in-person visit to a primary care provider, mental health provider or specialist who provides in-office evaluation, toxicology testing, and sends the patient to a pharmacy to obtain buprenorphine, which is then started at home. Continuation of care requires further in-person appointments on an interval basis.

'Higher' levels of OUD care offer additional structured support and in turn require additional commitment from patients: opioid treatment programmes require everyday attendance for at least a 90-day initial period; intensive outpatient programmes require at least 9 hours of structured programming each week per criteria set by the American Society of Addiction Medicine; partial
Summary box

What are the new findings?

- This novel clinical model of collaborative, integrated medical and behavioural healthcare that delivers biopsychosocial treatment of opioid use disorder (OUD) via telehealth-termed the Bicycle Health model-is an effective way to reach previously unengaged and unreached patients, as evidenced by $31 \%$ of Bicycle Health patients reporting no previous history of buprenorphine treatment. The three primary pillars to accomplish this goal include the following: patientcentred care, including chronic disease management and behavioural health; creation of tech-enabled systems and resources; and data-driven decision-making.

- The pharmacy finder tool enhances patient-centred care by enabling programme staff to identify pharmacies that are geographically near to the patient, tend to maintain buprenorphine in stock, and do not have a history of stigmatising or declining service to patients receiving tele-OUD treatment; this is evidenced by the ability of clinical support staff to successfully find medication in stock at a pharmacy convenient to the patient $75 \%$ of the time, compared with $40 \%$ prior to development of the pharmacy finder tool.

- Observable patterns demonstrate that retention rates in this novel model of biopsychosocial treatment of OUD via telehealth at 1 month and 3 months are appreciably higher than the industry average, as well as no-show rates that are significantly lower than industry average. 


\section{Summary box}

How might it impact on healthcare in the future?

- Results that demonstrate appreciably higher retention rates and lower no-show rates in a telehealth model of OUD treatment have far-reaching implications for access to care; this model may be replicated and adapted to increase access to OUD treatment, thereby curbing opioid morbidity and mortality.

hospitalisation programmes require at least 20 hours of structured programming each week; and residential and inpatient programmes offer continuous support. ${ }^{5}$ In-person treatment settings may offer alternative medications to buprenorphine, including methadone and naltrexone, which, for various reasons, are not feasible to offer via telehealth. It is notable that these settings in the OUD continuum of care are typically unforgiving of employment and family responsibilities that many treatment-seeking patients with OUD must balance. ${ }^{67}$

Additional deterrents to engagement in in-person treatment include stigmatisation of OUD care, as well as lack of buprenorphine waivered providers in highneed areas, particularly rural regions. ${ }^{8-10}$ According to a 2020 federal report, $36 \%$ of counties throughout the USA demonstrate high-need for buprenorphine services, yet patients in $56 \%$ of these counties lack adequate access to waivered providers. ${ }^{11}$ In fact, there exist 320 high-need counties in the USA with no buprenorphine waivered providers, resulting in significant disparities for patients desiring MOUD. ${ }^{11}$

The COVID-19 pandemic has resulted in a rapid shift to telehealth, including provision of OUD treatment via telehealth (also known as tele-OUD). In January 2020, the US Drug Enforcement Administration loosened remote prescribing restrictions under the Ryan Haight Act, ultimately lifting the requirement for an in-person visit prior to remote buprenorphine prescribing. ${ }^{12}$ These relaxed restrictions allow for improved access to buprenorphine treatment within the USA, and this improved access is evidenced by research from peer countries who have similarly relaxed buprenorphine restrictions in the past. ${ }^{13} 14$

Herein is a description of a novel clinical model of collaborative, integrated medical and behavioural healthcare that delivers technology-enabled biopsychosocial treatment of OUD via telehealth. This novel model is based on research that demonstrates the need for improved access to buprenorphine treatment as a means to curb opioid morbidity and mortality. ${ }^{15-17}$

\section{METHODS}

\section{Bicycle Health model}

Bicycle Health currently operates in 23 states, with five regional medical directors, as well as oversight by the Chief Medical Officer. ${ }^{18}$ The Bicycle Health model is organised into regional hubs, allowing medical providers, behavioural health coordinators and clinical support staff to become familiar with the community resources available to patients in each respective region, thereby optimising the ability to coordinate across healthcare systems and remotely address patients' social determinants of health (SDOH).

The Bicycle Health model of tele-OUD treatment includes three primary pillars:

- Patient-centred care, including chronic disease management and behavioural health.

- Creation of tech-enabled systems and resources.

- Data-driven decision-making.

To best enable and support patient-centred care, Bicycle Health has built various technology platforms to support patients' needs and treatment goals. In addition to advancing patient-centred care, research has shown that tech-enabled care can support clinical decision-making. ${ }^{19}$

No Institutional Review Board approval was required for this Early-Stage Innovation Report because developing the report did not involve interaction or intervention with human subjects or access to individually identifiable patient information; all data were collected in the course of routine clinical care and reviewed in aggregate.

\section{Patient treatment journey}

When patients first inquire with Bicycle Health about seeking treatment, they speak with an experienced enrolment coordinator who reviews basic demographics, medical and psychiatric history, and substance use history. Following the enrolment call, patients consent to treatment and are scheduled to meet with a medical provider within 24 hours. Medical providers include physicians, nurse practitioners and physician assistants with specialised training in addiction medicine. Patients interface with providers and staff via the Bicycle Health smartphone app. ${ }^{20}$

Providers conduct video calls with patients via the Zoom platform. Meeting privacy is protected by generating a unique Zoom event for each video visit with each individual patient, and by protecting each visit with a waiting room. In order to protect patient privacy and confidentiality, no telehealth visits are recorded. Additionally, none of the data on the Bicycle Health app is stored locally (ie, on phones or computers). Telehealth visits are encrypted using the Advanced Encryption Standard. ${ }^{21}$

Patients have access to tech and clinical support staff on various 'chat channels' within the Bicycle Health app. ${ }^{20}$ Clinical support staff can review provider notes and reinforce instructions to patients; direct patients to information about treatment expectations; (re) schedule appointments routinely or urgently; call pharmacies to provide support for prior authorisations; and share mental health and self-management resources. 
During the first medical provider visit, providers work with patients to develop individualised treatment plans based on medical, psychiatric and family histories, current and past substances used, history of buprenorphine, methadone, or naltrexone use, social supports and SDOH. If the provider diagnoses OUD based on DSM V criteria and performs a level of care assessment that qualifies the patient for the telehealth level of care, then patients typically receive a buprenorphine prescription after their first provider visit. ${ }^{22}$

Buprenorphine prescriptions are electronically sent by providers to patients' local pharmacies. Recent research demonstrates that nearly $30 \%$ of US pharmacies report limitations to filling buprenorphine prescriptions, and $20 \%$ of US pharmacies would not fill buprenorphine prescriptions entirely. ${ }^{23}$ To address these access issues, Bicycle Health built a pharmacy finder tool (on top of a dataset of all pharmacies in the USA) that allows clinical support staff to identify the pharmacy ahead of a patient's first visit that is geographically nearest to the patient, most likely to have buprenorphine in stock based on history, accepts the patient's insurance, and does not have a reported history of stigmatising or declining service to patients receiving tele-OUD treatment. Bicycle Health staff then call to confirm current buprenorphine stock, and for self-pay patients, also confirm that the pharmacy will accept prescription discount coupons. The patient thus has a seamless prescription-filling experience following their first visit, and if not, can access an after-hours on-call provider to troubleshoot filling difficulties.

The Bicycle Health model also uses its internally developed and evidence-based buprenorphine induction tool. This interactive tool guides patients through the induction process and is derived from the detailed plan established during a patient's first clinical visit. The induction tool pulls data directly from the provider's structured encounter note to customise the plan for each patient. After confirming data accuracy of the 14 variables pulled by the tool, including how long the patient should wait between each dose, the provider then sends the unique access link to the patient. The patient accesses the induction tool through their smartphone and answers questions to gauge where they are in the withdrawal process and when they can expect to take their first dose of buprenorphine, as shown in figure 1. Following the first dose of buprenorphine, patients complete self-assessment surveys and receive step-by-step instructions for follow-up doses based on timing and dose responses. Patients use this induction tool after every dose of buprenorphine until the second visit with their provider.

Next, the Bicycle Health urine drug test protocol has evolved significantly with the growth of the Bicycle Health patient population, ultimately reaching its current iteration. Each patient receives several urine test kits at a time, which are kept at home for immediate availability when a test is requested by their provider. Each CLIA-waived point-of-care cup tests

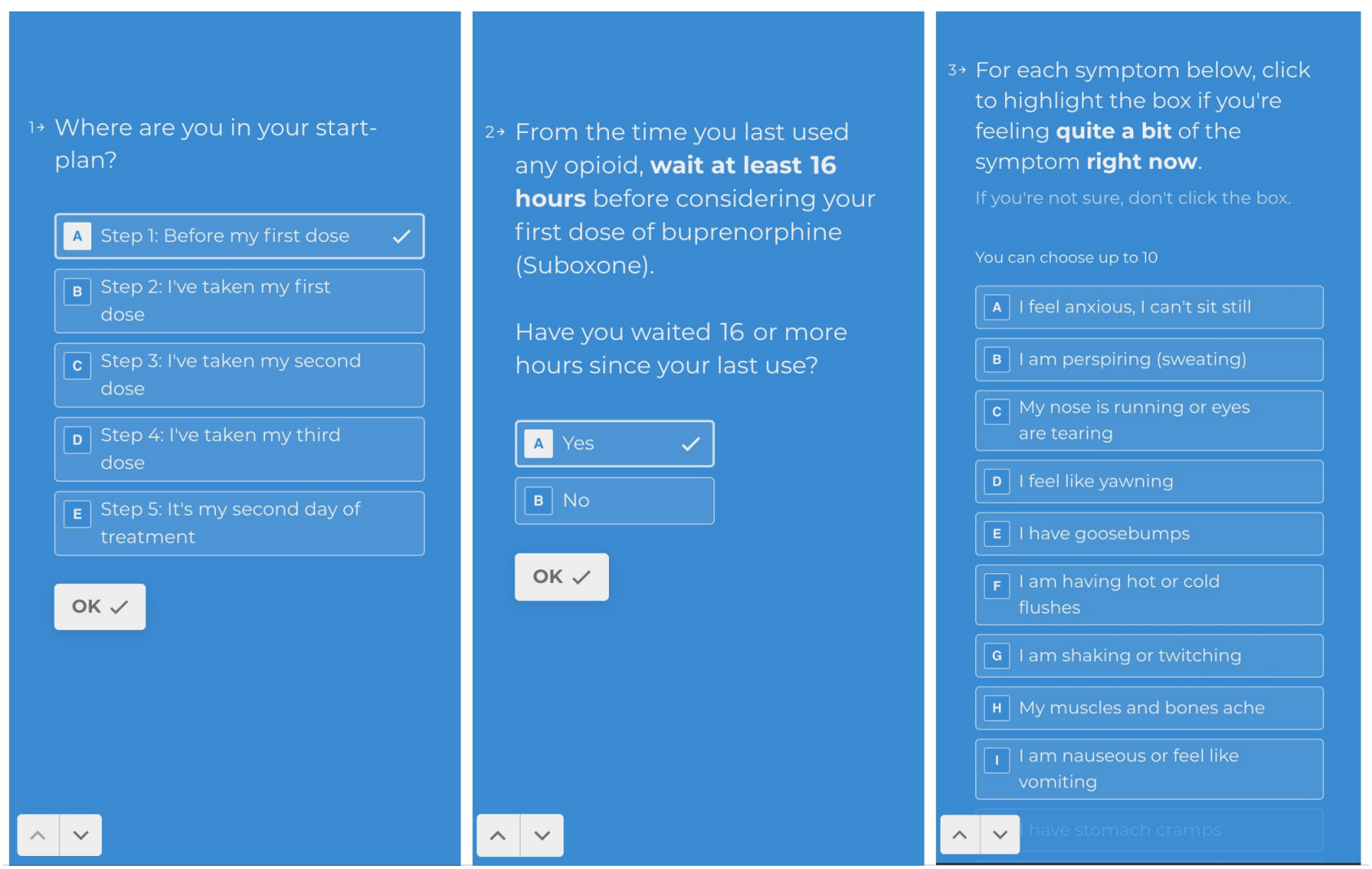

Figure 1 Example of the buprenorphine induction tool self-assessment symptom survey as viewed by patients. 
for 12 substances, and a separate fentanyl test dipstick is provided that is dipped into the cup in order to account for the rise in use of synthetic opioids. Since accountability depends on knowledge that a test can be performed at any time, urine testing intervals are ordered by providers based on clinical need, and exact test dates are randomly generated by an SMS prompting system:

- When prompted to complete a urine drug test, it should be done as soon as possible. If an ordered test is not completed by the next day, the delay is considered a sign of potential treatment concern.

- Providers may ask patients to complete a saliva drug screen during a video visit. This is requested randomly or for-cause and may be compared with recent urine results to assess sample source consistency.

- Providers may ask patients to complete a urine test at a nearby commercial lab, which allows for precise measurement of the type and amount of buprenorphine present in the sample.

Urine tests that are negative for buprenorphine may prompt the provider to order a confirmatory test at an in-person lab, as this may be indicative of buprenorphine diversion or the test being completed by someone other than the patient. Providers may also verify medication adherence through use of on-video medication counts.

Additionally, integrated behavioural health is a key part of the Bicycle Health model. ${ }^{24}$ An important component of such care includes a registry tool, which contains relevant data regarding patients' clinical status and barriers to care. ${ }^{25} 26$ Registries are organised by provider and include their respective panel of patients. Bicycle Health registry data includes, but is not limited to, the following: date of last patient appointment, future appointments, appointment no-shows, employment status, history of anxiety or depression, use of illicit substances, and history of intravenous drug use. Providers and behavioural health coordinators share patient panels, and behavioural health coordinators review each registry to help guide patient outreach and psychosocial interventions. Behavioural health coordinators are fully licensed clinicians in their respective fields (eg, licensed clinical social workers) and have addictions-focused expertise to further support the population that Bicycle Health serves.

Behavioural health services include virtual support groups, psychotherapy, and care coordination. Bicycle Health offers (at least) daily virtual support groups, led by licensed behavioural health clinicians and recovery coaches (staff members who have completed a recovery coach training programme and may have shared recovery experience). Behavioural health coordinators can also provide short-term targeted interventions via psychotherapy. All medical providers and behavioural health coordinators are trained in motivational interviewing.

\section{RESULTS}

Bicycle Health has served more than 10000 patients across 23 US states. The Bicycle Health patient population is $40.2 \%$ women and $59.8 \%$ men, with an age range of 18-77 years old. Further demographic information is limited at this time, though upcoming data collection revisions will provide more robust demographic data. At the time of enrolment, 31\% of Bicycle Health patients report no previous history of buprenorphine treatment.

Retention in treatment is the most commonly used measure in published literature for evaluating outcomes in OUD treatment. The retention rate for Bicycle Health patients at 30 days is $75 \%$, and the best retained population by payor is insured patients at $86 \%$ retention. The retention rate for Bicycle Health patients at 90 days is $59 \%$, and again, the best retained population by payor is insured patients at $80 \%$ retention. Industry average is challenging to approximate given wide inter-programme variability; one large claims data review finds $69 \%$ retention at 30 days and $44 \%$ retention at 90 days. ${ }^{27}$ The no-show rate for Bicycle Health patients is $9.5 \%$, compared with $23 \%$ average by a large systematic review. ${ }^{28}$ Approximately $70 \%$ of new patients are seen by a buprenorphinewaivered provider on the same or next business day as their initial enrolment outreach. Eighty-nine per cent of new patients receive provider-delivered motivational interviewing.

With particular respect to the pharmacy finder tool, implementation of this tool has appreciably reduced the time spent on locating buprenorphine stock and has helped to address financial and logistical barriers for patients in accessing buprenorphine. Prior to implementation of the pharmacy finder tool, clinical support staff spent extensive time locating a pharmacy for each new patient that would be able to fill their prescription same-day. Clinical support staff were only successful on the first call attempt in $40 \%$ of cases, would need to call between three to four pharmacies on average to locate stock, and were ultimately unsuccessful in finding stock same-day for $10 \%$ of patients. Following implementation of the pharmacy finder tool, clinical support staff successfully find stock on the first call attempt in $75 \%$ of cases, call 1.5 pharmacies on average, and are unsuccessful in finding stock same-day for less than $1 \%$ of patients. The pharmacy finder tool allows staff to filter for pharmacies within a specific distance range that each respective patient indicates is accessible to them.

\section{DISCUSSION}

Through development of a tech-enabled, collaborative model of integrated medical and behavioural healthcare that provides chronic disease management via telehealth, the Bicycle Health model is an innovative model of patient-centred tele-OUD treatment. To our knowledge, such a comprehensive model of 
biopsychosocial, tech-enabled treatment for OUD is the first of its kind.

An important limitation is the current inability to deploy in-app patient-facing questionnaires, screenings, and surveys to collect data regarding substance use, mood, relationships, healthcare utilisation, and quality of life, and to do this in a way that is useful for treating clinicians as well as for purposes of outcome evaluation. Currently, data collection efforts largely depend on direct provider interaction with patients and documentation, which is provider time-intensive. The technology capabilities required to utilise in-app questionnaires, screenings and surveys will soon be built by the Bicycle Health engineering team.

Bicycle Health continues to refine and optimise its model. An additional primary work-in-progress is development of a screening and active linkage process to address $\mathrm{SDOH}$, which has been a unique challenge in telehealth given the providers' frequent remoteness from local SDOH resources. Bicycle Health has also entered into a research partnership to evaluate the outcome of coupling a psychosocial needs screening questionnaire with a case management and referral system that uses coaches trained in motivational interviewing to target improved rates of successful referral completion when needs are identified.

Another unique challenge has been the task of creating avenues for patient investment and communitybuilding in their chosen telehealth home for OUD treatment. To this end, a team of staff members developed the Patient Advisory Council, which advises on patient-centred care and provides recommendations that respond to the unique needs and wishes of Bicycle Health patients and families.

\section{CONCLUSION}

OUD treatment has improved remarkably in recent decades, though many persons desiring indicated and necessary treatment remain unreached. The novel clinical model presented herein describes a collaborative, integrated medical and behavioural healthcare model that delivers tech-enabled biopsychosocial treatment of OUD via telehealth. The Bicycle Health model will continue to evolve via a patient-centred approach, of which the Patient Advisory Council will play a primary role. Continued efforts to increase access to buprenorphine treatment will help to curb opioid morbidity and mortality in the USA, and the Bicycle Health model is a novel example of such efforts. ${ }^{15-17}$

Acknowledgements The authors would like to acknowledge Nameera Faisal Akhtar and LuAnn Aakhus for their contributions to this article.

Contributors All coauthors have contributed substantially to the paper and have approved the version being submitted.

Funding The authors have not declared a specific grant for this research from any funding agency in the public, commercial or not-for-profit sectors.
Competing interests Authors RR, WG, LH, ET and SP are employed by Bicycle Health, Inc and receive income from Bicycle Health, Inc. BC is employed by Bicycle Health, Inc, and Bicycle Health Medical Group and receives income from Bicycle Health, Inc, and Bicycle Health Medical Group. WG, LH, ET, SP and BC have been granted equity in Bicycle Health, Inc.

Patient consent for publication Not applicable.

Ethics approval This study does not involve human participants.

Provenance and peer review Not commissioned; externally peer reviewed.

Open access This is an open access article distributed in accordance with the Creative Commons Attribution Non Commercial (CC BYNC 4.0) license, which permits others to distribute, remix, adapt, build upon this work non-commercially, and license their derivative works on different terms, provided the original work is properly cited, appropriate credit is given, any changes made indicated, and the use is non-commercial. See: http://creativecommons.org/ licenses/by-nc/4.0/.

\section{REFERENCES}

1 Ahmad FB, Rossen LM, Sutton P. Provisional drug overdose death counts. National center for health statistics. [cited 2021 Jul 25], 2021. Available: https://www.cdc.gov/nchs/nvss/vsrr/ drug-overdose-data.htm

2 U.S. Department of Health and Human Services, Substance Abuse and Mental Health Services Administration (SAMHSA)'s public online data analysis system (PDAS), National Survey on Drug Use and Health, 2020 (NSDUH2020-DS0001). [cited 2021 Dec 06]. Available: http://pdas. samhsa.gov/\#/survey/NSDUH-2020-DS0001/crosstab/? row $=$ OPMEDYR2\&column $=$ UDYR5OPI\&weight $=$ ANALWTQ1Q4_C\&run_chisq=false\&filter=UDYR5OPI $\%$ 3D1\&results_received $=$ true

3 Duncan A, Anderman J, Deseran T, et al. Monthly patient volumes of buprenorphine-waivered clinicians in the US. JAMA Netw Open 2020;3:e2014045.

4 American Society of Addiction Medicine. Public policy statement on the regulation of office-based opioid treatment. Rockville, MD; 2018 [cited 2021 Jul 25], 2018. Available: https://www.asam.org/docs/default-source/public-policystatements/statement-on-regulation-of-obot.pdf

5 Johns Hopkins Medicine Center for Substance Abuse Treatment and Research. Treatment settings. Baltimore, MD; n.d. [cited 2021 Jul 25]. Available: https://www. hopkinsmedicine.org/substance_abuse_center/treatment/ settings.html

6 Sulley S, Ndanga M. Inpatient opioid use disorder and social determinants of health: a nationwide analysis of the National inpatient sample (2012-2014 and 2016-2017). Cureus 2020;12:e11311.

7 Williams N, Bossert N, Chen Y, et al. Influence of social determinants of health and substance use characteristics on persons who use drugs pursuit of care for hepatitis $C$ virus infection. J Subst Abuse Treat 2019;102:33-9.

8 Tsai AC, Kiang MV, Barnett ML, et al. Stigma as a fundamental hindrance to the United States opioid overdose crisis response. PLoS Med 2019;16:e1002969.

9 Stone EM, Kennedy-Hendricks A, Barry CL, et al. The role of stigma in U.S. primary care physicians' treatment of opioid use disorder. Drug Alcohol Depend 2021;221:108627.

10 Cioe K, Biondi BE, Easly R, et al. A systematic review of patients' and providers' perspectives of medications for treatment of opioid use disorder. J Subst Abuse Treat 2020;119:108146. 
11 U.S. Department of Health and Human Services (HHS) Office of Inspector General. Geographic disparities affect access to buprenorphine services for opioid use disorder. Washington, DC; [cited 2021 Jul 25], 2020. Available: https://oig.hhs.gov/ oei/reports/oei-12-17-00240.asp

12 U.S. Department of Justice Drug Enforcement Administration (DEA): Diversion Control Division. COVID-19 information page. Springfield, VA; [cited $2021 \mathrm{Jul}$ 25], 2020. Available: https://www.deadiversion.usdoj.gov/coronavirus.html

13 Woodruff AE, Tomanovich M, Beletsky L, et al. Dismantling buprenorphine policy can provide more comprehensive addiction treatment. NAM Perspect 2019;2019. doi:10.31478/201909a. [Epub ahead of print: 0909 2019].

14 Fatseas M, Auriacombe M. Why buprenorphine is so successful in treating opiate addiction in France. Curr Psychiatry Rep 2007;9:358-64.

15 Khatri U, Davis CS, Krawczyk N. These key telehealth policy changes would improve buprenorphine access while advancing health equity. Health Affairs Blog. [cited 2021 Jul 25], 2020. Available: https://www.healthaffairs.org/do/10.1377/ hblog20200910.498716/full/

16 The Pew Charitable Trusts. Policies should promote access to buprenorphine for opioid use disorder: state and federal leaders can eliminate barriers, boost treatment. Philadelphia, PA; [cited 2021 Jul 25], 2021. Available: https://www. pewtrusts.org/en/research-and-analysis/issue-briefs/2021/05/ policies-should-promote-access-to-buprenorphine-for-opioiduse-disorder

17 Davis CS, Samuels EA. Continuing increased access to buprenorphine in the United States via telemedicine after COVID-19. Int J Drug Policy 2021;93:102905.

18 Bicycle Health. Boston, MA; 2021 [cited 2021 Jul 25]. Available: https://www.bicyclehealth.com/

19 Hallgren KA, Bauer AM, Atkins DC. Digital technology and clinical decision making in depression treatment: current findings and future opportunities. Depress Anxiety 2017;34:494-501.

20 App Store Preview. Bicycle Health. [cited 2021 Jul 25], 2021. Available: https://apps.apple.com/us/app/bicycle-health/ id1560854220

21 Dworkin MJ, Barker EB, Nechvatal JR. Advanced Encryption Standard (AES). U.S. Department of Commerce National Institute of Standards and Technology. Gaithersburg, MD; [cited 2021 Jul 25], 2001. Available: https://www.nist.gov/ publications/advanced-encryption-standard-aes

22 Centers for Disease Control and Prevention (CDC). Assessing and addressing opioid use disorder (OUD). Atlanta, GA; n.d. [cited 2021 Jul 25]. Available: https://www.cdc.gov/ drugoverdose/training/oud/accessible/index.html

23 Kazerouni NJ, Irwin AN, Levander XA, et al. Pharmacy-related buprenorphine access barriers: an audit of pharmacies in counties with a high opioid overdose burden. Drug Alcohol Depend 2021;224:108729.

24 Lim CT, Fulwiler CE, Carson NJ. Promoting population behavioral health in a safety-net health system during the COVID-19 pandemic. Psychiatr Serv 2021:appips202000546.

25 Pop B, Fetica B, Blaga ML, et al. The role of medical registries, potential applications and limitations. Med Pharm Rep 2019;92:7-14.

26 Hoque DME, Kumari V, Hoque M, et al. Impact of clinical registries on quality of patient care and clinical outcomes: a systematic review. PLoS One 2017;12:e0183667.

27 Morgan JR, Schackman BR, Leff JA, et al. Injectable naltrexone, oral naltrexone, and buprenorphine utilization and discontinuation among individuals treated for opioid use disorder in a United States commercially insured population. $J$ Subst Abuse Treat 2018;85:90-6.

28 Dantas LF, Fleck JL, Cyrino Oliveira FL, et al. No-shows in appointment scheduling - a systematic literature review. Health Policy 2018;122:412-21. 